\title{
Qualitative behavior of a smoking model
}

\author{
Qamar Din ${ }^{1 *}$, Muhammad Ozair², Takasar Hussain² and Umer Saeed ${ }^{3}$
}

"Correspondence: qamar.sms@gmail.com

'Department of Mathematics, The University of Poonch Rawalakot,

Rawalakot, 12350, Pakistan

Full list of author information is

available at the end of the article

\begin{abstract}
We study the qualitative behavior of a smoking model in which the population is divided into five classes, that is, non-smokers, smokers, people who temporarily quit smoking, people who permanently quit smoking, and people who are associated with illness due to smoking. The global asymptotic stability of the unique positive equilibrium point is presented. More precisely, a graph-theoretic method is used to prove the global stability of the unique positive equilibrium point.
\end{abstract}

Keywords: epidemic model; local and global stability; Lyapunov function; graph-theoretic method

\section{Introduction}

Smoking is one of the main causes of health problems and continues to be one of the world's most significant health challenges. It is the leading cause of preventable death, and it is estimated to kill more than 5 million people worldwide each year, and this number is expected to grow. According to the World Health Organization report on the global tobacco epidemic [1], tobacco use kills or disables many people in their most productive years, which denies families their primary wage-earners, consumes family budgets, raises the cost of health care and hinders economic development. Smoking or tobacco is a known or probable cause of deaths from cancers of the oral cavity, larynx, lung, esophagus, bladder, pancreas, renal pelvis, stomach, and cervix. Smoking is also a cause of heart disease, strokes, peripheral vascular diseases, chronic obstructive lung diseases, and other respiratory diseases, and low-birth weight babies [2].

Exerting more pressure for immediate intervention and public policy making are the reported increases in tobacco and drug use by young people. The Surgeon General reports that in 1991 the average age when smokers tried a cigarette for the first time was 14.5 years, and the average age when they became daily smokers was 17.7 years. The observed trend from 1975 to 1992 of relevant data confirm that adolescence is the primary time during which tobacco use develops [3]. Smoking among adolescents is also connected to social factors. Adolescents whose families and friends smoke are more likely to start smoking earlier than their counterparts.

The enormous public health burden related with smoking urges one to study the dynamics of smoking in a community, intended at determining realistic methods for preventing this habit. Mathematical modeling has been used extensively to address questions of public health importance, dating back to the seminal work of Bernoulli (on modeling the dy-

(c) 2016 Din et al. This article is distributed under the terms of the Creative Commons Attribution 4.0 International License (http://creativecommons.org/licenses/by/4.0/), which permits unrestricted use, distribution, and reproduction in any medium, provided you give appropriate credit to the original author(s) and the source, provide a link to the Creative Commons license, and indicate if changes were made. 
namics of smallpox) in 1760 [4], Kermack and McKendrick [5-7] and those reported in more recent literature (such as in [8-10] and the references therein); not much has been analyzed in terms of the mathematical modeling of human social behavior.

In 2000, Castillo-Garsow et al. [11] for the first time proposed a simple mathematical model for giving up smoking. They consider a system with a total constant population which is divided into three classes: potential smokers, that is, people who do not smoke yet but might become smokers in the future (P), smokers (S), and people (former smokers) who have quit smoking permanently $(\mathrm{Q})$. Sharomi and Gumel developed mathematical models by introducing mild and chain classes [12]. In their work they presented the development and public health impact of smoking-related illnesses. Zaman [13] extended the work of Castillo-Garsow et al. [12] and developed a model taking into account the occasional smokers compartment in the given smoking model and presented its qualitative behavior.

The model presented in [14] studied the dynamics of a given smoking model. In this work, the population was divided in four subclasses: potential smokers, occasional smokers, smokers, and quit smokers. In this paper, we modified this model by including the following features:

- Smokers who temporarily quit smoking and return back to smoking class.

- A class of smokers associated with some illness.

- The additional death rate of the smoking-related illness class.

The rest of the paper is organized as follows. In Section 2, we present a formulation of the modified mathematical model. The existence of endemic equilibrium and its local stability is presented in Section 3. In Section 4, we use a graph-theoretic method to show the global stability of the endemic equilibrium. An estimation of the parameters and numerical results are discussed in Section 5. Finally, we give our conclusion.

\section{Formulation of model}

The schematic diagram of the proposed model is shown in Figure 1.

The description of the variables and the parameters is given in Table 1.

The analytical expression of the above model, shown in the flow diagram, is

$$
\begin{aligned}
& \frac{d P}{d t}=\alpha-\beta f(P, S)-\gamma P, \\
& \frac{d S}{d t}=\beta f(P, S)-(\gamma+\delta+\epsilon) S+\zeta X, \\
& \frac{d X}{d t}=\delta(1-\eta) S-(\gamma+\zeta) X, \\
& \frac{d Y}{d t}=\delta \eta S-\gamma Y,
\end{aligned}
$$

Figure 1 Flow diagram of smoking model.

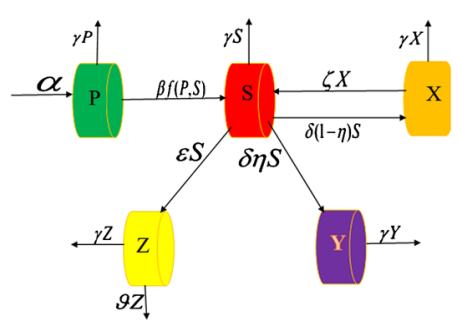


Table 1 Description of variables and parameters

\begin{tabular}{ll}
\hline Parameter & Description \\
\hline$P$ & Potential smokers \\
$S$ & Smokers \\
$X$ & Smokers who temporarily quit smoking \\
$Y$ & Smokers who permanently quit smoking \\
$Z$ & Class of smokers associated with some illness \\
$\alpha$ & The recruitment rate of the non-smoking (potential smoking) class from the larger embed- \\
$\beta$ & ding population \\
$\gamma$ & The transmission rate of non-smokers into smoking class \\
$\delta(1-\eta)$ & The natural death rate \\
$\delta \eta$ & The rate at which smokers temporarily quit smoking \\
$\epsilon$ & The rate at which smokers permanently quit smoking. It is assumed that $0<\eta<1$ \\
$\zeta$ & Developing rate of smoking-related illness of the smokers \\
$\vartheta$ & The rate at which temporarily quit smokers return back to smoking class \\
\hline
\end{tabular}

$$
\frac{d Z}{d t}=\epsilon S-(\gamma+\vartheta) Z
$$

Our aim in this paper is to discuss the qualitative behavior of above model by taking

$$
f(P, S)=\sqrt{P S}
$$

In this case the system (2.1)-(2.5) can be rewritten as

$$
\begin{aligned}
& \frac{d P}{d t}=\alpha-\beta \sqrt{P S}-\gamma P, \\
& \frac{d S}{d t}=\beta \sqrt{P S}-(\gamma+\delta+\epsilon) S+\zeta X, \\
& \frac{d X}{d t}=\delta(1-\eta) S-(\gamma+\zeta) X, \\
& \frac{d Y}{d t}=\delta \eta S-\gamma Y, \\
& \frac{d Z}{d t}=\epsilon S-(\gamma+\vartheta) Z .
\end{aligned}
$$

Assume that system (2.6)-(2.10) has non-negative initial conditions, then every solution $(P(t), S(t), X(t), Y(t), Z(t))$ of (2.6)-(2.10) has the positivity property, i.e., $P(t) \geq 0, S(t) \geq 0$, $X(t) \geq 0, Y(t) \geq 0$, and $Z(t) \geq 0$.

Consider the following equation:

$$
N(t)=P(t)+S(t)+X(t)+Y(t)+Z(t) .
$$

Hence, we obtain

$$
\frac{d N}{d t}=\alpha-\gamma N-Z \vartheta
$$

Then it follows that

$$
\frac{d N}{d t} \leq \alpha-\gamma N
$$


with $N(0)=P(0)+S(0)+X(0)+Y(0)+Z(0)$. Then from (2.11), it follows that

$$
\lim _{t \rightarrow \infty} \sup N(t) \leq \frac{\alpha}{\gamma}
$$

\section{Endemic equilibrium and its local stability}

An endemic equilibrium is one in which there are variables corresponding to the presence of the disease in the population that are non-zero. Local stability of an equilibrium point means that if we put the system somewhere near the point then it will move to the equilibrium point in some time. Global stability means that the system will come to the equilibrium point from any possible starting point (i.e., there is no 'nearby' condition). In an even more physical interpretation, if an endemic equilibrium is locally stable then all epidemiological situations not so much different from the given stable equilibrium will (with time) evolve to (or transform into) the equilibrium point. Also it means that the equilibria are stable to small perturbations, i.e., if we push the situation a bit away from the equilibrium point then the situation will return on its own (from the physicist's point of view, it means that the equilibrium may be a stable situation in real life, because the real world always is somewhat noisy). Global stability of an equilibrium point in this case may be described as 'the inevitable fate of the epidemic process regardless of its starting situation'. But the caveat should be considered that this 'inevitability' holds as long as the world strictly follows the underlying mathematical model of the epidemic process.

Let $E=\left(P^{*}, S^{*}, X^{*}, Y^{*}, Z^{*}\right)$ be an equilibrium point of (2.6)-(2.10), then

$$
\begin{aligned}
P^{*} & =\frac{\alpha\left(\gamma^{2}+\gamma(\delta+\zeta+\epsilon)+\zeta(\delta \eta+\epsilon)\right)}{\beta^{2}(\gamma+\zeta)+\gamma\left(\gamma^{2}+\gamma(\delta+\zeta+\epsilon)+\zeta(\delta \eta+\epsilon)\right)}, \\
S^{*} & =\frac{\alpha \beta^{2}(\gamma+\zeta)^{2}}{\left(\gamma^{2}+\gamma(\delta+\zeta+\epsilon)+\zeta(\delta \eta+\epsilon)\right)\left(\beta^{2}(\gamma+\zeta)+\gamma\left(\gamma^{2}+\gamma(\delta+\zeta+\epsilon)+\zeta(\delta \eta+\epsilon)\right)\right)}, \\
X^{*} & =\frac{\alpha \beta^{2} \delta(1-\eta)(\gamma+\zeta)}{\left(\gamma^{2}+\gamma(\delta+\zeta+\epsilon)+\zeta(\delta \eta+\epsilon)\right)\left(\beta^{2}(\gamma+\zeta)+\gamma\left(\gamma^{2}+\gamma(\delta+\zeta+\epsilon)+\zeta(\delta \eta+\epsilon)\right)\right)}, \\
Y^{*} & =\frac{\alpha \beta^{2} \delta \eta(\gamma+\zeta)^{2}}{\gamma\left(\gamma^{2}+\gamma(\delta+\zeta+\epsilon)+\zeta(\delta \eta+\epsilon)\right)\left(\beta^{2}(\gamma+\zeta)+\gamma\left(\gamma^{2}+\gamma(\delta+\zeta+\epsilon)+\zeta(\delta \eta+\epsilon)\right)\right)}, \\
Z^{*} & =\frac{\alpha \beta^{2} \epsilon(\gamma+\zeta)^{2}}{(\gamma+\vartheta)\left(\gamma^{2}+\gamma(\delta+\zeta+\epsilon)+\zeta(\delta \eta+\epsilon)\right)\left(\beta^{2}(\gamma+\zeta)+\gamma\left(\gamma^{2}+\gamma(\delta+\zeta+\epsilon)+\zeta(\delta \eta+\epsilon)\right)\right)} .
\end{aligned}
$$

Hence, $\left(P^{*}, S^{*}, X^{*}, Y^{*}, Z^{*}\right)$ is the unique positive equilibrium point of (2.6)-(2.10).

The feasible region $\Gamma=\left\{(P, S, X, Y, Z) \in \mathbb{R}_{+}^{5}: P+S+X+Y+Z \leq \frac{\alpha}{\gamma}\right\}$ is positively invariant with respect to (2.6)-(2.10). Furthermore, $\left(P^{*}, S^{*}, X^{*}, Y^{*}, Z^{*}\right) \in \operatorname{int}(\Gamma)[15]$.

Theorem 3.1 The unique positive equilibrium point of (2.6)-(2.10) is locally asymptotically stable. 
Proof The Jacobian matrix $J_{M}\left(P^{*}, S^{*}, X^{*}, Y^{*}, Z^{*}\right)$ associated with $\left(P^{*}, S^{*}, X^{*}, Y^{*}, Z^{*}\right)$ is given by

$$
\begin{aligned}
& J_{M}\left(P^{*}, S^{*}, X^{*}, Y^{*}, Z^{*}\right) \\
& =\left[\begin{array}{ccccc}
-\frac{\beta \sqrt{S^{*}}}{2 \sqrt{P^{*}}}-\gamma & -\frac{\beta \sqrt{P^{*}}}{2 \sqrt{S^{*}}} & 0 & 0 & 0 \\
\frac{\beta \sqrt{S^{*}}}{2 \sqrt{P^{*}}} & \frac{\beta \sqrt{P^{*}}}{2 \sqrt{S^{*}}}-(\gamma+\delta+\epsilon) & \zeta & 0 & 0 \\
0 & \delta(1-\eta) & -(\gamma+\zeta) & 0 & 0 \\
0 & \delta \eta & 0 & -\gamma & 0 \\
0 & \epsilon & 0 & 0 & -(\gamma+\vartheta)
\end{array}\right] .
\end{aligned}
$$

Furthermore, the characteristic polynomial of $J_{M}\left(P^{*}, S^{*}, X^{*}, Y^{*}, Z^{*}\right)$ about $\left(P^{*}, S^{*}\right.$, $\left.X^{*}, Y^{*}, Z^{*}\right)$ is given by

$$
C(\lambda)=(\lambda+\gamma)(\lambda+\gamma+\vartheta) Q(\lambda)
$$

where

$$
Q(\gamma)=\lambda^{3}+a \lambda^{2}+b \lambda+c
$$

with

$$
\begin{aligned}
a= & 3 \gamma+\delta+\zeta+\frac{\beta\left(S^{*}-P^{*}\right)}{2 \sqrt{P^{*} S^{*}}}+\epsilon \\
b= & \left(P^{*}\left(2 S^{*}\left(3 \gamma^{2}+2 \gamma(\delta+\zeta+\epsilon)+\zeta(\delta \eta+\epsilon)\right)-\beta(2 \gamma+\zeta) \sqrt{P^{*} S^{*}}\right)\right. \\
& \left.+\beta S^{*} \sqrt{P^{*} S^{*}}(2 \gamma+\delta+\zeta+\epsilon)\right) \\
& /\left(2 P^{*} S^{*}\right)
\end{aligned}
$$

and

$$
\begin{aligned}
c= & \left(\beta S^{*} \sqrt{P^{*} S^{*}}\left(\gamma^{2}+\gamma(\delta+\zeta+\epsilon)+\zeta(\delta \eta+\epsilon)\right)\right. \\
& \left.+\gamma P^{*}\left(2 S^{*}\left(\gamma^{2}+\gamma(\delta+\zeta+\epsilon)+\zeta(\delta \eta+\epsilon)\right)-\beta(\gamma+\zeta) \sqrt{P^{*} S^{*}}\right)\right) \\
& /\left(2 P^{*} S^{*}\right) .
\end{aligned}
$$

It follows from (3.1) that $\lambda_{1}=-\gamma$ and $\lambda_{2}=-(\gamma+\vartheta)$ are two eigenvalues of the Jacobian matrix $J_{M}\left(P^{*}, S^{*}, X^{*}, Y^{*}, Z^{*}\right)$, and its remaining eigenvalues are the roots of (3.1). Furthermore, after some tedious calculations, one can show that

$$
a>0, \quad b>0, \quad c>0, \quad a b>c .
$$

Hence, according to the Hurwitz criterion, the unique positive equilibrium point of (2.6)(2.10) has local asymptotical stability. 


\section{Global stability}

In order to prove the global stability of unique endemic equilibrium, we use a graphtheoretic method. One can find this method in [16, 17]. Arguing as in [15], we will study some basic results related to the graph-theoretic method.

A directed graph, or digraph, $G$, consists of a set of vertices $V(G)$, a set of $\operatorname{arcs} A(G)$, and a function which assigns each $\operatorname{arc} A$ an ordered pair of vertices $(i, j)$. We call $i$ the tail of $A$, $j$ the head of $A$, and $i, j$ the ends of $A$. If there is an arc with tail $i$ and head $j$, then we let $(i, j)$ denote such an arc, and we say that this arc is directed from $i$ to $j$. The indegree $d_{G}^{-}(i)$ of vertex $i$ is the number of $\operatorname{arcs}(l, i), l \in V$. The outdegree $d_{G}^{+}(i)$ of vertex $i$ is the number of $\operatorname{arcs}(i, l), l \in V$. Moreover, assume that $H$ is a spanning set of $G$ having the same vertex sets, then we call $H$ a subdigraph of $G$. If we assign a positive weight to each arc, then the digraph $G$ is said to be weighted. The weight $W(H)$ of a subdigraph $H$ is the product of the weights on all its arcs.

A rooted graph, or simply a tree, is a subdigraph, denoted by $T$ of $G$, and it is a single connected component in which the indegree of one vertex is zero, whereas each of the remaining vertices has indegree one. On the other hand, a directed path $P$ is a subdigraph with distinct vertices denoted by $i_{1}, i_{2}, \ldots, i_{k}$ such that its arcs are of the form $\left(i_{m}, i_{m+1}\right)$, where $m=1,2, \ldots, k-1$. Moreover, we denote a directed cycle by $C$, and it is the subdigraph obtained from such a path $P$ by adding the $\operatorname{arc}\left(i_{k}, i_{1}\right)$.

Let $G$ be a weighted digraph having $n$ vertices. Next, we consider an $n \times n$ weighted matrix denoted by $M=\left(a_{i j}\right)$ such that $a_{i j}>0$ equal to the weight of arc $(j, i)$ if it exists and 0 otherwise. We denote such a weighted digraph by $(G, M)$. A digraph $G$ is strongly connected if, for any pair of distinct vertices $i, j$, there exists a directed path from $i$ to $j$. A weighted digraph $(G, M)$ is strongly connected if and only if the weight matrix $M$ is irreducible. The Laplacian matrix $L=\left(l_{i j}\right)$ of $(G, M)$ is defined as

$$
l_{i j}= \begin{cases}-a_{i j}, & i \neq j, \\ \sum_{i \neq k} a_{i k}, & i=k .\end{cases}
$$

Lemma 4.1 (Kirchhoff's matrix tree theorem) For $n \geq 2$, assume that $c_{i}$ is the cofactor of $l_{i i}$ in $L$. Then $c_{i}=\sum_{T \in T_{i}} W(T), i=1,2, \ldots, n$, where $T_{i}$ is the set of all spanning trees $T$ of $(G, M)$ that are rooted at vertex $i$. Moreover, if $(G, M)$ is strongly connected, then $c_{i}>0$ for $1 \leq i \leq n$.

Moreover, arguing as in $[15,18]$, we have the following results.

Lemma 4.2 Let $c_{i}$ be as given in Lemma 4.1. Then $\sum_{i, j=1}^{n} c_{i} a_{i j} F_{i}(X)=\sum_{i, j=1}^{n} c_{i} a_{i j} F_{j}(X)$, where $F_{i}(X)$ is a collection of functions with $X=\left(x_{1}, \ldots, x_{m}\right) \in \mathbb{R}^{m}$, and $1 \leq i \leq n$.

Lemma 4.3 [15] Let $c_{i}$ be as given in Lemma 4.1. Let $a_{i j}>0$, and $d_{G}^{+}(j)=1$ for some $1 \leq$ $i, j \leq n$, then $c_{i} a_{i j}=\sum_{k=1}^{n} c_{j} a_{j k}$.

Theorem 4.4 The unique positive equilibrium point of system (2.6)-(2.10) is globally asymptotically stable. 
Proof Using the graph-theoretic method developed in [15], first we construct the Lyapunov function $V(t)$. For this purpose, we set

$$
\begin{array}{ll}
V_{1}=P-P^{*}-P^{*} \ln \frac{P}{P^{*}}, & V_{2}=S-S^{*}-S^{*} \ln \frac{S}{S^{*}}, \\
V_{3}=X-X^{*}-X^{*} \ln \frac{X}{X^{*}}, & V_{4}=Y-Y^{*}-Y^{*} \ln \frac{Y}{Y^{*}},
\end{array}
$$

and

$$
V_{5}=Z-Z^{*}-Z^{*} \ln \frac{Z}{Z^{*}} .
$$

Differentiation w.r.t. $t$ gives

$$
\begin{aligned}
V_{1}^{\prime}= & \left(1-\frac{P^{*}}{P}\right) P^{\prime}, \\
V_{1}^{\prime}= & \left(1-\frac{P^{*}}{P}\right)(\alpha-\beta \sqrt{P S}-\gamma P), \\
V_{1}^{\prime}= & \beta\left(1-\frac{P^{*}}{P}\right)\left(\sqrt{P^{*} S^{*}}-\sqrt{P S}\right)+\gamma\left(1-\frac{P^{*}}{P}\right)\left(P^{*}-P\right), \\
V_{1}^{\prime}= & \beta \sqrt{P^{*} S^{*}}\left(1-\frac{P^{*}}{P}\right)\left(1-\frac{\sqrt{P S}}{\sqrt{P^{*} S^{*}}}\right)-\gamma \frac{\left(P-P^{*}\right)^{2}}{P}, \\
V_{1}^{\prime}= & -\gamma \frac{\left(P-P^{*}\right)^{2}}{P} \\
& +\beta \sqrt{P^{*} S^{*}}\left(\sqrt{\frac{P^{*}}{P}} \sqrt{\frac{S}{S^{*}}}-\ln \sqrt{\frac{P^{*}}{P}} \sqrt{\frac{S}{S^{*}}}+\ln \sqrt{\frac{P^{*}}{P}} \sqrt{\frac{S}{S^{*}}}+1-\frac{P^{*}}{P}-\frac{\sqrt{P S}}{\sqrt{P^{*} S^{*}}}\right), \\
V_{1}^{\prime} \leq & \beta \sqrt{P^{*} S^{*}}\left(\sqrt{\frac{P^{*}}{P}} \sqrt{\frac{S}{S^{*}}}-\ln \sqrt{\frac{P^{*}}{P}} \sqrt{\frac{S}{S^{*}}}+\ln \sqrt{\frac{P S}{P^{*} S^{*}}}-\frac{\sqrt{P S}}{\sqrt{P^{*} S^{*}}}\right) \\
= & : a_{12} G_{12} .
\end{aligned}
$$

Similarly we obtain

$$
\begin{aligned}
V_{2}^{\prime}= & \left(1-\frac{S^{*}}{S}\right) S^{\prime}, \\
V_{2}^{\prime}= & \left(1-\frac{S^{*}}{S}\right)(\beta \sqrt{P S}-(\gamma+\delta+\epsilon) S+\zeta X), \\
V_{2}^{\prime} \leq & \beta \sqrt{P^{*} S^{*}}\left(\sqrt{\frac{P S}{P^{*} S^{*}}}-\ln \sqrt{\frac{P S}{P^{*} S^{*}}}+\ln \sqrt{\frac{P}{P^{*}}}-\sqrt{\frac{P}{P^{*}}}-\sqrt{\frac{S^{*}}{S}}+\ln \sqrt{\frac{S^{*}}{S}}\right) \\
& +\zeta X^{*}\left(\frac{X}{X^{*}}-\ln \frac{X}{X^{*}}-\frac{X}{X^{*}} \frac{S^{*}}{S}+\ln \frac{X}{X^{*}} \frac{S^{*}}{S}\right) \\
= & : a_{23} G_{23}+a_{25} G_{25}, \\
V_{3}^{\prime}= & \left(1-\frac{X^{*}}{X}\right) X^{\prime}, \\
V_{3}^{\prime}= & \left(1-\frac{X^{*}}{X}\right)(\delta(1-\eta) S-(\gamma+\zeta) X),
\end{aligned}
$$




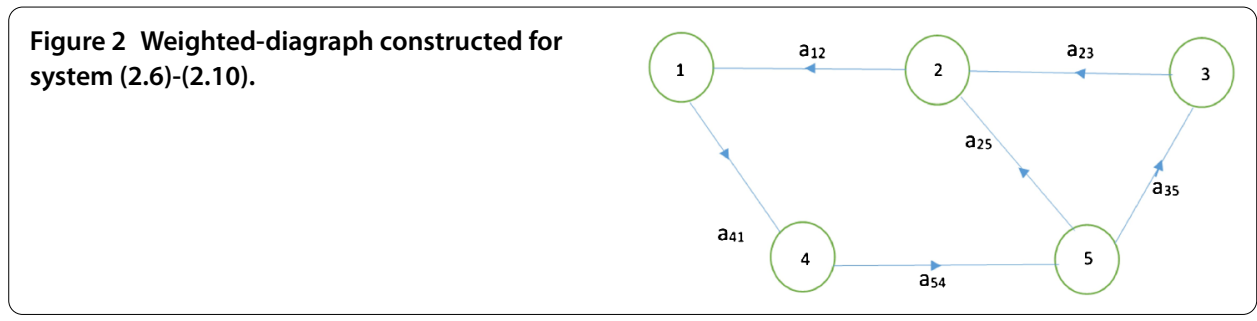

$$
\begin{aligned}
V_{3}^{\prime} & \leq \delta(1-\eta) S^{*}\left[\frac{S}{S^{*}}-\frac{S X^{*}}{S^{*} X}+\ln \frac{S X^{*}}{S^{*} X}-\ln \frac{S}{S^{*}}\right] \\
& =: a_{35} G_{35}, \\
V_{4}^{\prime} & =\left(1-\frac{Y^{*}}{Y}\right) Y^{\prime}, \\
V_{4}^{\prime} & =\left(1-\frac{Y^{*}}{Y}\right)[\delta \eta S-\gamma Y], \\
V_{4}^{\prime} & \leq \delta \eta S^{*}\left[\frac{S}{S^{*}}-\ln \frac{S}{S^{*}}-\frac{S}{S^{*}} \frac{Y^{*}}{Y}+\ln \frac{S}{S^{*}} \frac{Y^{*}}{Y}\right] \\
& =: a_{41} G_{41}, \\
V_{5}^{\prime} & =\left(1-\frac{Z^{*}}{Z}\right) Z^{\prime}, \\
V_{5}^{\prime} & =\left(1-\frac{Z^{*}}{Z}\right)[\epsilon S-(\gamma+\vartheta) Z], \\
V_{5}^{\prime} & \leq \epsilon S^{*}\left[\frac{S}{S^{*}}-\ln \frac{S}{S^{*}}-\frac{S}{S^{*}} \frac{Z^{*}}{Z}+\ln \frac{S}{S^{*}} \frac{Z^{*}}{Z}\right] \\
& =: a_{54} G_{54} .
\end{aligned}
$$

The associated weighted digraph has five vertices and two cycles (see Figure 2). Along each cycle $G_{12}+G_{41}+G_{54}+G_{25}=0$ and $G_{12}+G_{41}+G_{54}+G_{35}+G_{23}=0$. By Theorem 3.5 in [15], there exists $c_{i}, 1 \leq i \leq 5$, such that $V=\sum_{i=1}^{5} c_{i} V_{i}$ is a Lyapunov function for (2.6)-(2.10). The relations between $c_{i}$ 's can be derived from Theorems 3.3 and 3.4 of [15]: $d^{+}(1)=1$ implies $c_{4} a_{41}=c_{1} a_{12}, d^{+}(2)=1$ implies $c_{1} a_{12}=c_{2}\left(a_{23}+a_{25}\right), d^{+}(3)=1$ implies $c_{2} a_{23}=c_{3} a_{35}$, and $d^{+}(4)=1$ implies $c_{5} a_{54}=c_{4} a_{41}$. Therefore $V=\sum_{i=1}^{5} c_{i} V_{i}=$ $V_{1}+\frac{\beta \sqrt{P^{*} S^{*}}}{\left(\beta \sqrt{\left.P^{*} S^{*}+\zeta X^{*}\right)}\right.} V_{2}+\frac{\beta P^{*} S^{*}}{\delta(1-\eta)\left(\beta \sqrt{\left.P^{*} S^{*}+\zeta X^{*}\right)}\right.} V_{3}+\frac{\beta}{\delta \eta} \sqrt{\frac{P^{*}}{S^{*}}} V_{4}+\frac{\beta}{\epsilon} \sqrt{\frac{P^{*}}{S^{*}}} V_{5}$. We can easily verify that $\{E\}$ is the only invariant set in $\operatorname{int}(\Omega)$, where $V^{\prime}=0$, therefore, $E$ is globally asymptotically stable in $\operatorname{int}(\Omega)$.

Physically the global stability of unique endemic equilibrium means that if smokers continue to spread the disease in the population, whatever the initial population will be, ultimately the population approaches a constant level. The constant level means that the transition in different classes of the population stops.

\section{Numerical simulations and discussion}

In this section the model is solved by using a Runge-Kutta fourth order scheme. The values of some of the parameters in the model are dictated by reality, e.g. the death rates of the 


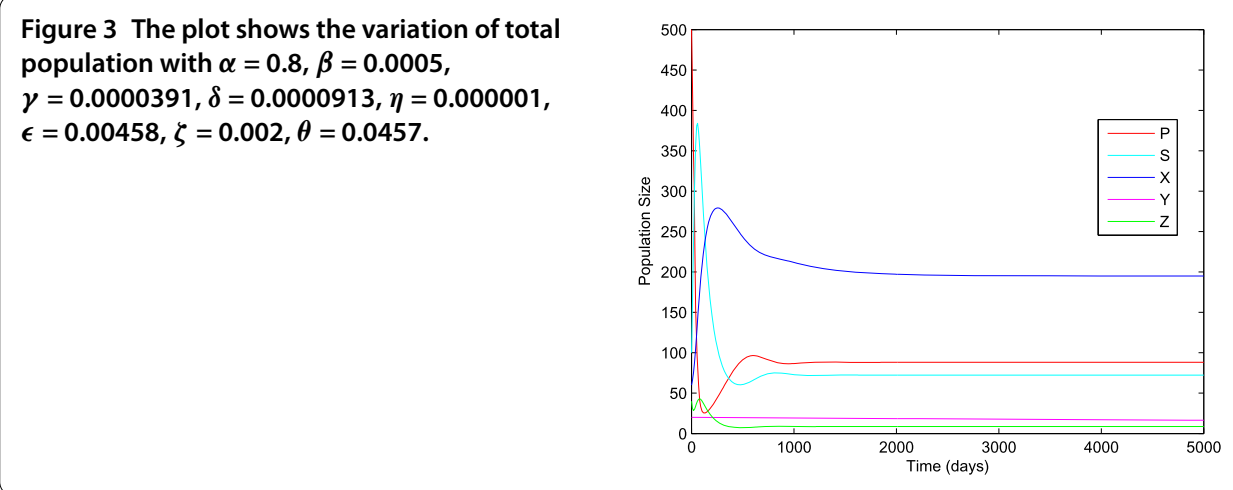

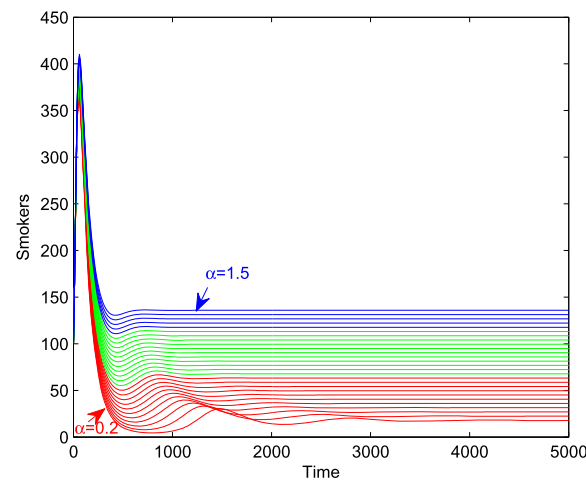

(a)

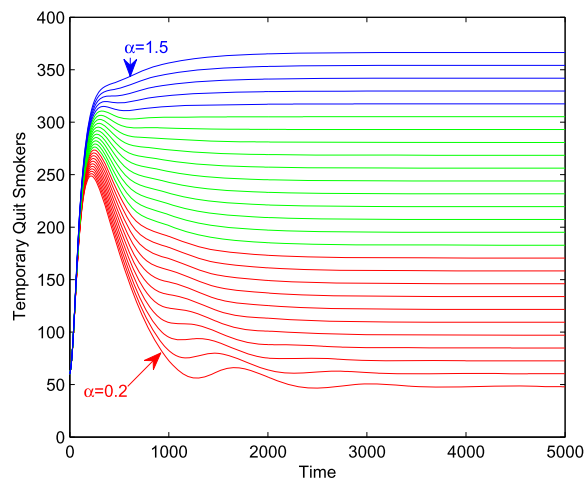

(b)

Figure 4 Endemic levels of $S$ and $X$ for different values of $\alpha$.

humans and the rate at which smokers quit smoking. The values of the parameters determined by nature are $\gamma=0.0000391$ per day, corresponding to a life expectancy of humans of 70 years and $\delta=0.0000913$ per day, corresponding to an average duration of smoking of 30 years. The variation of the total population is shown in Figure 3. From this figure we see that the population approaches a unique endemic equilibrium whenever the reproductive number exceeds unity and the value of $R_{0}$ is 93.33. It means that when disease persists and the smokers continue to infect potential smokers even then the population approaches the constant level.

The endemic level of smokers and temporarily quit smokers can be decreased or increased by decreasing or increasing the value of the different parameters. Figures 4, 5, 6, 7,8 , and 9 represent endemic levels of smokers and temporary quit smokers for different values of the parameter. We observe that increasing the values of $\delta$, the endemic level of temporary quit smokers increases. It means that the smokers enter in the temporary quit smoking class at the first moment. It can also be observed that by increasing the factor $\eta$ the endemic level of temporary quit smokers decreases. These observations suggest that enhancing the awareness in the population about the menace of smoking can motivate the smokers and temporary quit smokers to enter in the permanently quit smoking class. In this way we can reduce this endemic disease. 


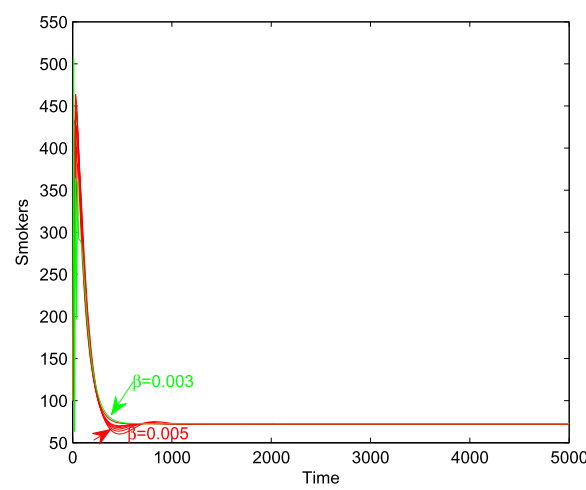

(a)

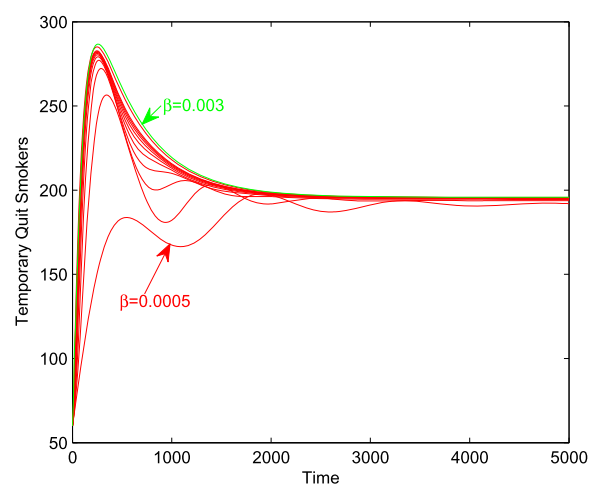

(b)

Figure 5 Endemic levels of $S$ and $X$ for different values of $\beta$.

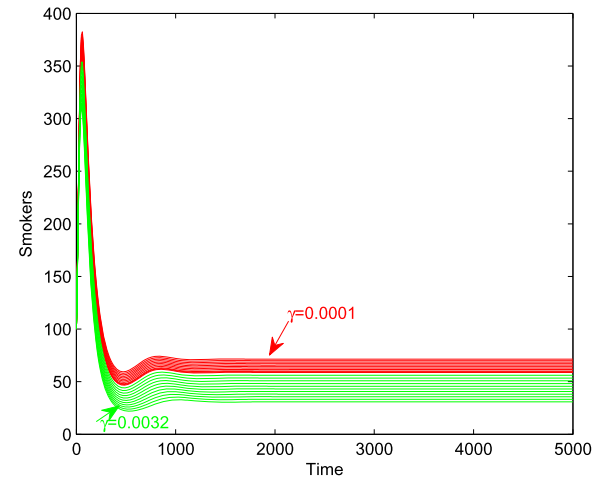

(a)

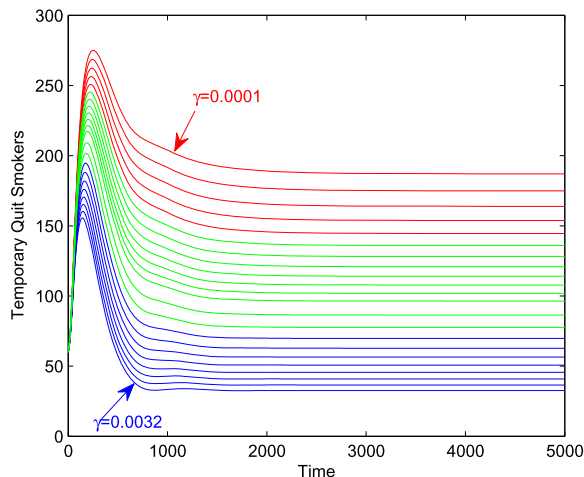

(b)

Figure 6 Endemic levels of $S$ and $X$ for different values of $\gamma$.

\section{Conclusion}

In this work we modified the model by taking into account smokers who temporarily quit smoking, a class of smokers associated with some illness, and the additional death rate of the smoking-related illness class. We first established the local stability of the endemic equilibrium by using a Hurwitz criterion and then used a graph-theoretic approach to prove the global stability. The typical solution of the system is obtained and showed that the population approaches the endemic level. It has also been observed that the endemic level of smokers and temporarily quit smokers is decreased by increasing the factor $\eta$. It means that the endemic level of smokers could be decreased through education and treatment campaigns to minimize the number of smokers and maximize the number of quit smokers in a community. The main objective of dynamical systems theory is to predict the global behavior of a system based on the knowledge of its present state. An approach to this problem consists of determining the possible global behavior of the system and determining which conditions on real parameters lead to this long-term behavior. In the case of nonlinear dynamical systems, it is very crucial to discuss the global behavior of the system. Particularly, the condition for global stability in population biology is a very 


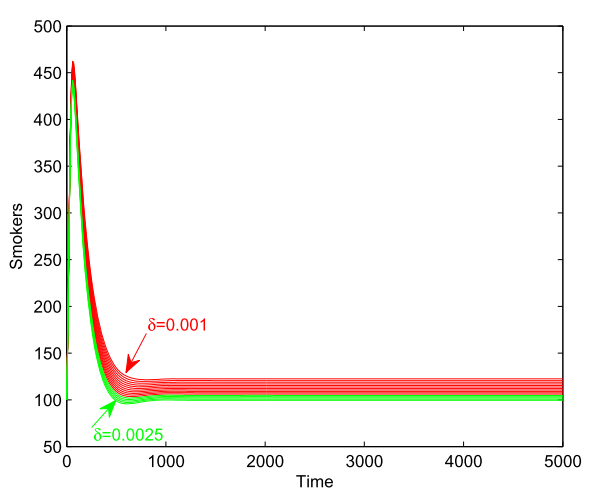

(a)

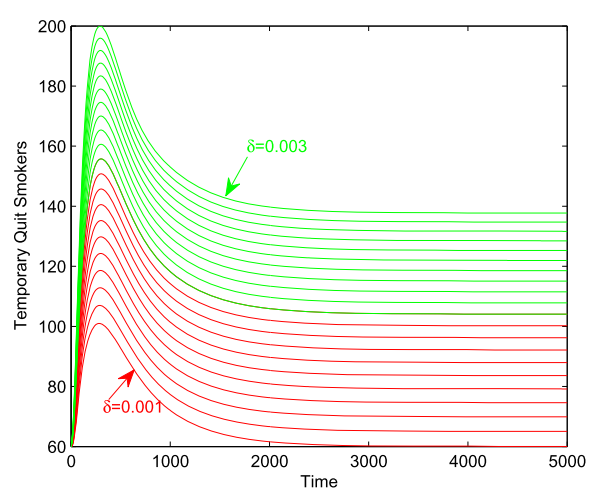

(b)

Figure 7 Endemic levels of $S$ and $X$ for different values of $\delta$.

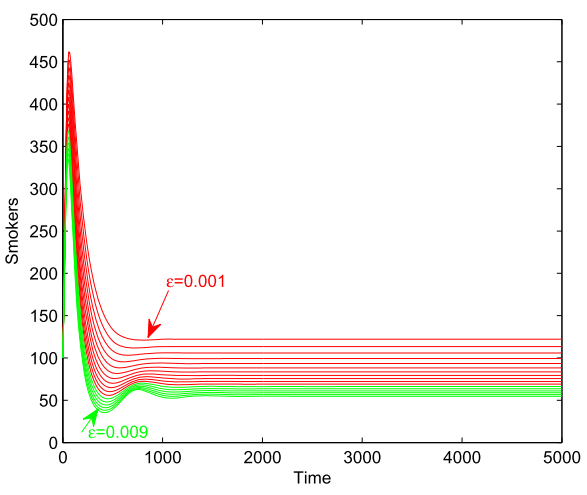

(a)

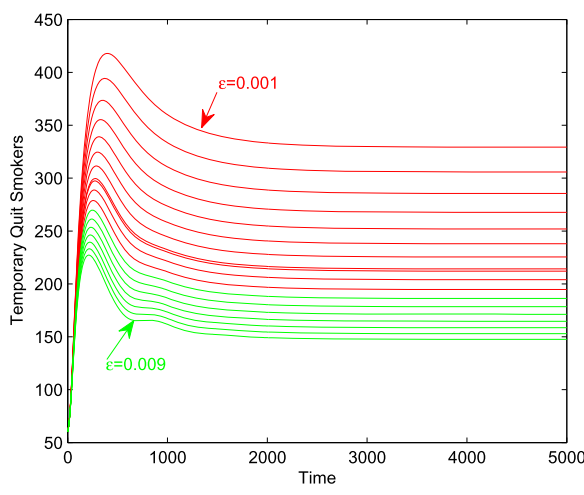

(b)

Figure 8 Endemic levels of $S$ and $X$ for different values of $\epsilon$.

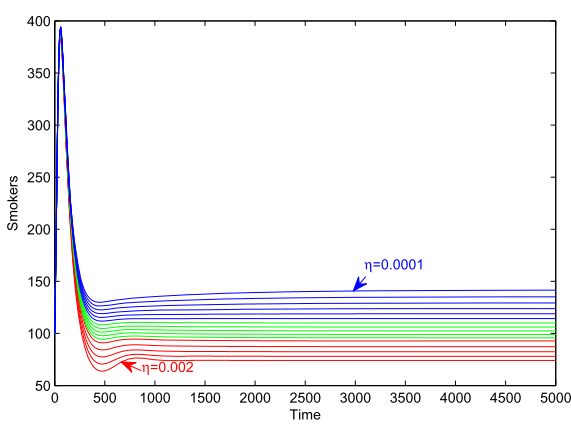

(a)

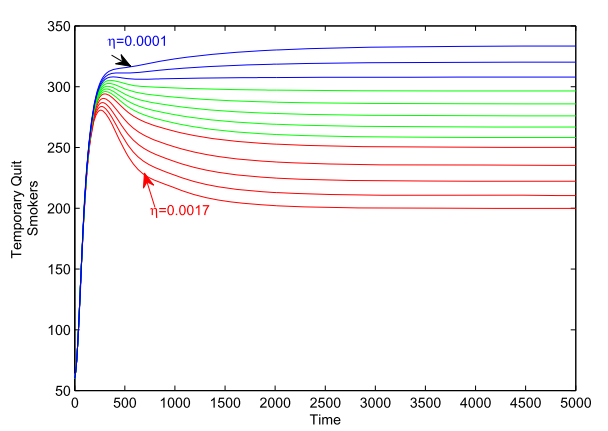

(b)

Figure 9 Endemic levels of $S$ and $X$ for different values of $\eta$. 
interesting mathematical problem. Therefore, it is very important to find conditions which may guarantee the global stability of the unique positive equilibrium point of the given system. In the paper, we prove the conditions for the global asymptotic stability of the unique positive equilibrium point for system (2.6)-(2.10).

\section{Competing interests}

The authors declare that they have no competing interests.

\section{Authors' contributions}

All authors carried out the proofs of the main results and approved the final manuscript.

\section{Author details}

'Department of Mathematics, The University of Poonch Rawalakot, Rawalakot, 12350, Pakistan. ${ }^{2}$ Department of Mathematics, COMSATS Institute of Information Technology, Attock, Pakistan. ${ }^{3}$ NUST Institute of Civil Engineering, National University of Sciences and Technology, Sector H-12, Islamabad, Pakistan.

\section{Acknowledgements}

The authors thank the main editor and anonymous referees for their valuable comments and suggestions leading to improvement of this paper. This work was partially supported by the Higher Education Commission of Pakistan.

Received: 31 July 2015 Accepted: 30 March 2016 Published online: 05 April 2016

\section{References}

1. World, $\mathrm{H}$ : Organization report on the global tobacco epidemic

http://whqlibdoc.who.int/publications/2009/9789241563918_eng_full.pdf (2009)

2. Lahrouz, A, Omari, L, Kiouach, D, Belmaati, A: Deterministic and stochastic stability of a mathematical model of smoking. Stat. Probab. Lett. 81(8), 1276-1284 (2011)

3. Johnston, LD, O'Malley, PM, Bachman, JG: Smoking, Drinking, and Illicit Drug Use Among American Secondary School Students, College Students, and Young Adults, 1975-1992. Volume 2: College Students and Young Adults. US Department of Health and Human Services, National Institutes of Health, Bethesda (1992)

4. Bernoulli, D: Essai d'une nouvelle analyse de la mortalite causee par la petite verole, et des avantages de l'inoculation pour la prevenir. Histoire de l'Academie Royale des Sciences, memoire de mathematiques et de physique, Paris, 1-45 (1760)

5. Kermark, WO, Mckendrick, AG: Contributions to the mathematical theory of epidemics. Part I. Proc. R. Soc. Lond. Ser. A 115, 700-721 (1927)

6. Kermark, WO, Mckendrick, AG: Contributions to the mathematical theory of epidemics. Part II. Proc. R. Soc. Lond. Ser. A 138, 55-83 (1932)

7. Kermark, WO, Mckendrick, AG: Contributions to the mathematical theory of epidemics. Part III. Proc. R. Soc. Lond. Ser. B 141, 94-112 (1933)

8. Anderson, RM, May, RM (eds.): Population Biology of Infectious Diseases. Springer, Berlin (1982)

9. Anderson, RM, May, RM: Infectious Diseases of Humans. Oxford University Press, London (1991)

10. Brauer, F, Castillo-Chavez, C: Mathematical Models in Population Biology and Epidemiology. Springer, Berlin (2001)

11. Castillo-Garsow, C, Jordan-Salivia, G, Herrera, AR: Mathematical models for the dynamics of tobacco use, recovery, and relapse. Technical Report Series BU-1505-M, Cornell University, Ithaca, NY, USA (1997)

12. Sharomi, O, Gumel, AB: Curtailing smoking dynamics: a mathematical modeling approach. Appl. Math. Comput. 195(2), 475-499 (2008)

13. Zaman, G: Qualitative behavior of giving up smoking model. Bull. Malays. Math. Soc. 34(2), $403-415$ (2011)

14. Zeb, A, Zaman, G, Momani, S: Square-root dynamics of a giving up smoking model. Appl. Math. Model. 37, 5326-5334 (2013)

15. Shuai, Z, Driessche, PVD: Global stability of infectious disease models using Lyapunov functions. SIAM J. Appl. Math. 73(4), 1513-1532 (2013)

16. Harary, F: Graph Theory. Addison-Wesley, Reading (1969)

17. West, DB: Introduction to Graph Theory. Prentice Hall, Upper Saddle River (1996)

18. Li, MY, Shuai, Z: Global-stability problems for coupled systems of differential equations on networks. J. Differ. Equ. 248, $1-20(2010)$ 